\section{Structure of rhodopsin}

\begin{abstract}
Two-dimensional crystals of rhodopsin were studied to determine the arrangement of the transmembrane alpha helices. A combination of electron cryo-microscopy, image processing and electron crystallography was used to extract amplitudes and phases from images, and a three-dimensional map to a resolution of $7.5 \AA$ A was calculated. Density peaks for all seven transmembrane helices were observed and the helix axes for all seven helices could be estimated. Near the intracellular side, which interacts with the $G$ protein transducin, we observed three layers of helices arranged differently from bacteriorhodopsin. The arrangement opens up towards the extracellular side forming a cavity that serves as the binding pocket for the retinal. This cavity is closed towards the intracellular side by the long and highly tilted helix 3 , and must be closed towards the extracellular side by the loop linking helices 4 and 5 that is linked by a disulphide bridge to the extracellular end of helix 3 .
\end{abstract}

Key words Vision, Rhodopsin, Membrane protein, Helices, Two-dimensional crystals, Electron microscopy

\footnotetext{
G.F.X. Schertler MRC Laboratory of Molecular Biology Hills Road Cambridge $\mathrm{CB} 22 \mathrm{QH}$, UK

Tel: +44 (0)1223402291

Fax: $+44(0) 1223213556$ e-mail:

gfx@mrc-Imb.cam.ac.uk
}

most GPCRs, rhodopsin is present in very high concentrations in the rod outer segment of the photoreceptor cell. The 11-cis retinal is covalently bound via a protonated Schiff base to lysine 196 and this helps to keep the dark noise in the visual system down since it acts like a covalently bound antagonist keeping the photoreceptor in a non-signalling conformation. In addition, rhodopsin has a rigid extracellular domain that also might help to reduce dark noise. Rhodopsin is one of the most stable and detergent-tolerant GPCRs known and it can be isolated from retinas in large quantities, by lectin affinity chromatography, making it an ideal candidate for structural investigations.

Electron crystallography of two-dimensional crystals of rhodopsin

Comparison of electron crystallography with $X$-ray crystallography

In principle NMR, $X$-ray crystallography or electron microscopy could be used to determine the structure of a membrane protein. However, so far no-one has been able to determine the structure of a membrane protein larger than 35 kDa by solution NMR. Only solid-state NMR has some prospect to do so in the future. In contrast, several structures of membrane proteins have been solved to near-atomic resolution with X-ray crystallography., ${ }^{4,5}$ Similarly with electron crystallography, the structures of several important membrane proteins were solved to a similar resolution., In addition, quite a number of low-resolution structures of membrane proteins have been obtained using electron microscopy.

The main differences between electron crystallography and X-ray crystallography are a much stronger interaction of electrons with matter and the possibility of using electromagnetic lenses to obtain images. Because of the strong interaction of electrons with matter, much smaller and thinner samples such as a large single protein complex, ${ }^{8}$ or a two-dimensional crystal consisting of a monolayer of protein molecules can be studied. However, this strong interaction also causes damage to the specimens and, therefore, cryotechniques and low-dose strategies are essential for the imaging of beam-sensitive biological specimens. Using electron microscopy we cannot only record diffraction patterns and 
measure amplitudes as with $\mathrm{X}$-rays, but also record images from which we can extract phases as well as amplitudes by Fourier analysis. Furthermore, computer processing of images allows us to remove some of the disorder present in the crystal lattice. In this way we can obtain important structural information even from nonideal two-dimensional crystals.

\section{Specimens for high-resolution electron microscopy: single particles, helical arrangements and two-dimensional crystals}

Why do we need a two-dimensional crystal to obtain a structure from rhodopsin by electron crystallography? Electron microscopy in combination with single particle image processing is able to give us structures from proteins and protein complexes of more than $300 \mathrm{kDa}$ since the signal obtained from a low-dose image of a large particle is strong enough to determine the orientation of the particle on a micrograph. ${ }^{8}$ After alignment, images from thousands of particles are averaged and a three-dimensional reconstruction of the protein structure is calculated. However, the molecular weight of rhodopsin and other GPCRs is too low for this approach. With present imaging techniques an image of a single rhodopsin molecule does not contain enough information for the alignment that is essential for averaging.

We therefore need a large regular and rigid aggregate to obtain structural information for a medium-sized protein of 30-200 kDa. Helical arrangements have been successfully used to obtain the structure of the nicotinic acetylcholine receptor ${ }^{9}$ and of the Ca-ATPase of the sarcoplasmic reticulum. ${ }^{10}$

Quite a few membrane protein structures were obtained from two-dimensional crystals, in which the membrane protein is arranged in a regular array that can be described by two lattice vectors: $\mathbf{a}$ and $\mathbf{b}$. These twodimensional cystals are held together by protein-protein, lipid-lipid and lipid-protein interactions.

\section{Image processing}

The low dose of electrons used to take electron micrographs of biological samples means that the image taken from a single molecule without averaging is noisy. Only the average of many well-aligned images gives us structural detail. Therefore, the aim of image processing is to achieve the best possible alignment before averaging. In this way image processing can improve the signal-to-noise ratio. ${ }^{11}$

In an image of a non-ideal two-dimensional crystal there are two major sources of distortions. One is the disorder in the crystal, which can be induced partially by the supporting carbon film and partially by the mechanical stress induced by the shrinkage during freezing. The second source of distortions is the imaging system of the electron microscope itself. With image processing both types of image distortion can be corrected for. A small reference area of the best part of the crystal is used to find distortion vectors for every part of the crystal that are then used to correct the image. In the corrected image all unit cells are more accurately aligned and therefore we can obtain an improved signalto-noise ratio for amplitudes and phases after the Fourier analysis. In this way we can extract higher-resolution data from non-ideal two-dimensional crystals.

\section{Preparation of two-dimensional rhodopsin crystals Crystallisation of membrane proteins}

In some rare cases membrane proteins form crystals in the cell membrane. Purple membrane is an example of a natural two-dimensional crystal, whereas halorhodopsin and gap junctions form crystalline arrays in the cell when they are overexpressed to a very high level. Since the density of the membrane is increased because of the high concentration of the protein in a two-dimensional crystal, density gradients can be used to obtain a purified crystalline sample. However, most membrane proteins do not form crystals spontaneously in the membrane. In this case the membrane must be solubilised in a buffer containing detergent and the protein must be purified by chromatography or density centrifugation in the presence of detergent.

\section{Reconstitution experiments with lipids}

Most chromatography steps are only partially delipidating; affinity steps or density gradients in particular, do not remove lipid efficiently. Therefore twodimensional crystals can sometimes be obtained by removing the detergent without adding any additional lipid. After several chromatography steps, often fewer than 5 lipids per molecule are associated with the protein and additional lipid has to be added before the detergent is removed by dialysis or by adsorption to biobeads. The aim of a reconstitution experiment is to form a large single membrane with enough protein incorporated so that crystallisation is induced. ${ }^{12}$

\section{Crystal induction by selective extraction of membranes}

From the rod outer segments in the retina we can isolate the disc membranes using Ficoll density flotation. These photoreceptor membranes are unique in that they contain a very high concentration of rhodopsin and about 70 lipids per protein, ${ }^{13}$ but the protein is still highly mobile in this lipid bilayer that contains many multiple unsaturated fatty acid side-chains. Corless and co-workers ${ }^{14}$ discovered that Tween detergents induce the formation of two-dimensional crystals of frog rhodopsin in the disc membranes. We were able to improve the reproducibility and crystal quality by optimising the Tween-to-protein ratio, the $\mathrm{pH}$ and the buffer composition. A further improvement was made by combining different Tween detergents. ${ }^{15}$ 

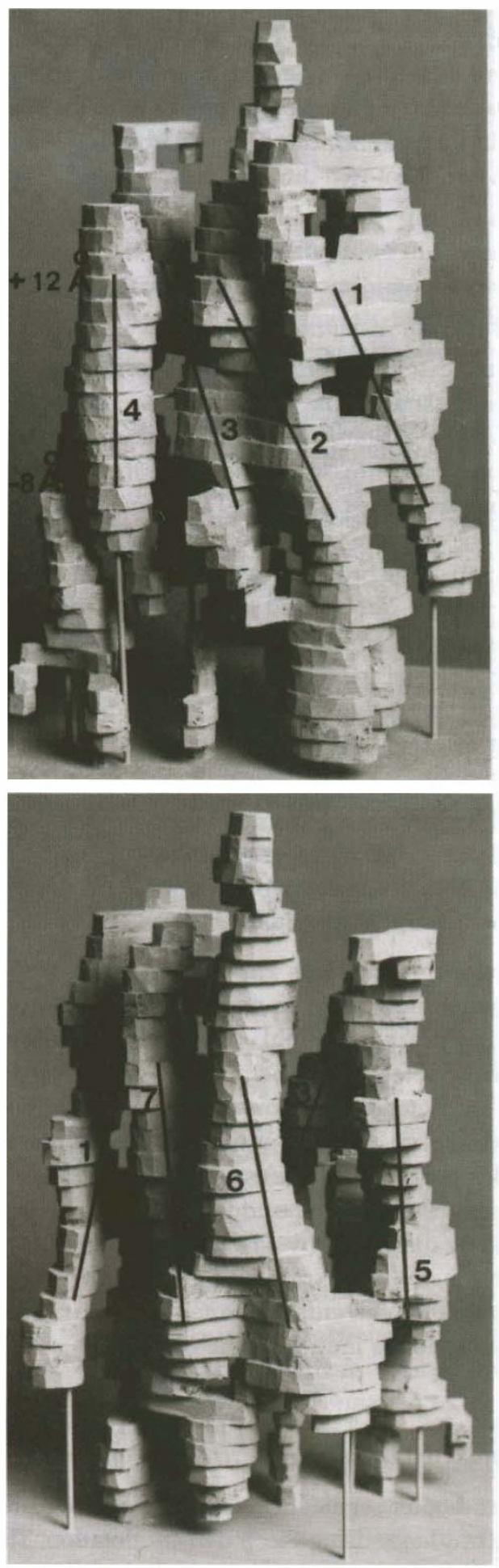

(A)
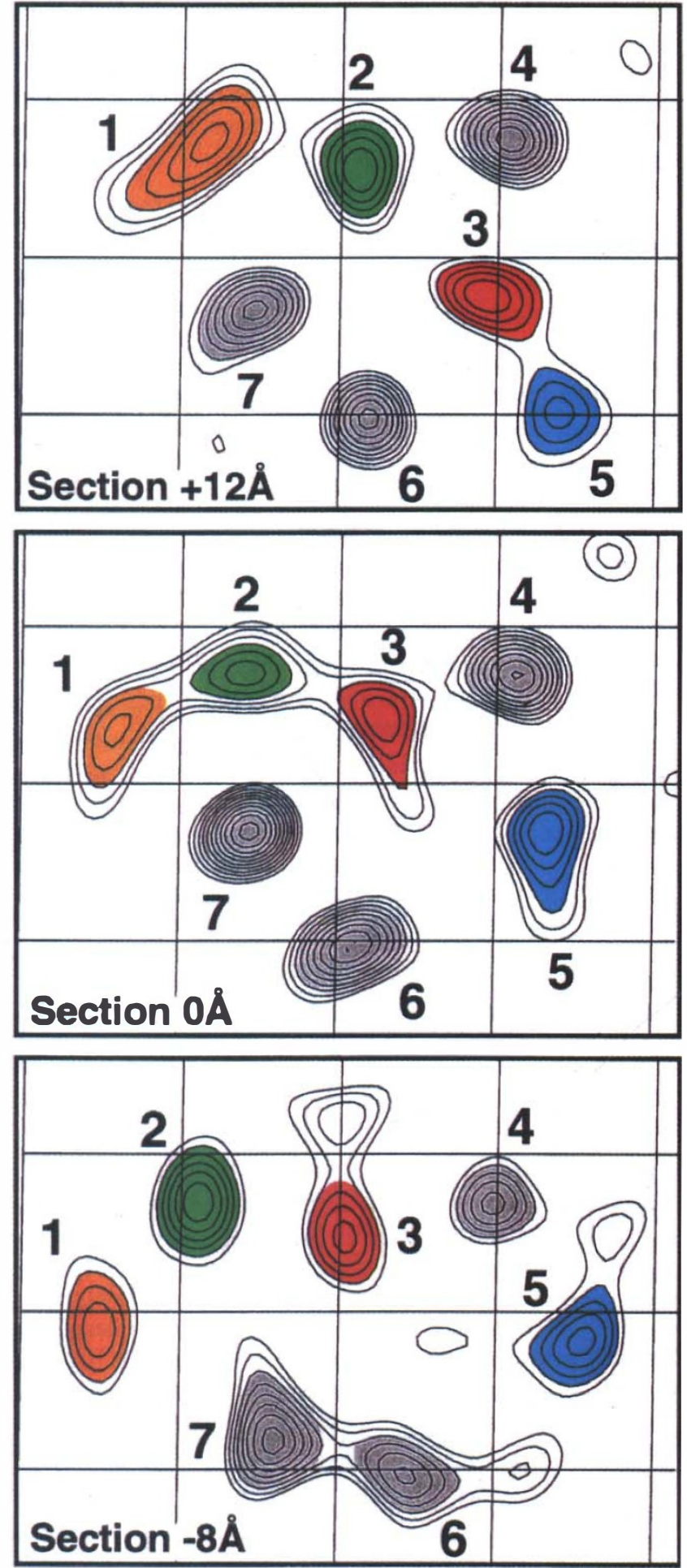

(B)

Fig. 1. The seven helices in the rhodopsin structure. (A) Structure of frog rhodopsin obtained by electron cryo-microscopy. ${ }^{19}$ Two views of a solid model of the rhodopsin map are shown: viewed from helix 2 towards helix 6 (above) and viewed from helix 6 towards helix 3 (below). The model was constructed from 33 contour sections $2 \AA$ apart. The cytoplasmic side is at the top and the intradiscal or extracellular side is at the bottom. The central sections of the seven transmembrane helices are marked with lines starting at section $+12 \AA$ at the top and ending at section $-8 \AA$. The corresponding sections are shown in (B). The peaks representing the seven helices are interpreted according to the sequence assignment ${ }^{17}$ to the projection map of rhodopsin..$^{16}$ Adapted from Unger et al. ${ }^{19}(B)$ Three slices through the best part of the density map of rhodopsin are shown. ${ }^{19}$ In each of these sections peaks can be seen for each of the seven transmembrane helices. The section closer to the cytoplasmic side is at $\mathrm{z}=+12 \AA$ from the centre and the last section at $\mathrm{z}=-8 \AA$ from the centre of the map. The least tilted helices 4,6 and 7 are coloured grey and the most tilted in four different colours. The grid spacing is $10 \AA$ with lines parallel to the $\boldsymbol{a}$ and $\boldsymbol{b}$ axes ( $+\boldsymbol{b}$ is horizontal to the right and $+\boldsymbol{a}$ points towards the bottom of the figure). Adapted from Unger et al. ${ }^{19}$ 


\section{The arrangement of alpha-helices in the membrane}

Direct evidence for the arrangement of the seven alphahelices was obtained from a $9 \AA$ projection map of bovine rhodopsin. ${ }^{16}$ Structural constraints obtained from a comparison of G-protein-coupled-receptor sequences were used to assign the seven hydrophobic stretches in the sequence to features in the projection map. ${ }^{17}$ A lowresolution three-dimensional structure of bovine rhodopsin ${ }^{18}$ and two projection structures of frog rhodopsin ${ }^{15}$ confirmed the position of the three least tilted helices 4, 6 and 7. A more elongated peak of density for helix 5 indicated that it is tilted or bent. ${ }^{15,18}$ Helices 1 , 2 and 3 were not resolved.

The extraction of frog rod cell membranes with Tween- 80 resulted in the formation of two-dimensional crystals with p2 symmetry. ${ }^{15}$ The crystals were better ordered than two-dimensional crystals that had been obtained previously by reconstitution of detergentpurified bovine rhodopsin in synthetic lipids. ${ }^{16}$ The three-dimensional map had an effective resolution of 7.5 $\AA$ in the membrane plane and $16.5 \AA$ normal to it. ${ }^{19}$

A single rhodopsin molecule in this map has planar dimensions of $28 \AA \times 39 \AA$. The molecule appears $64 \AA$ high at the chosen contour level of Fig. 1A, but because of the low vertical resolution this is only an approximation. In contoured cross-sections taken parallel to the membrane plane the clearest features are close to the middle of the membrane. The central sections of the seven transmembrane helices are marked in Fig. 1A with lines starting at section $+12 \AA$ and ending at section $-8 \AA$. This part of the map is contained within the hydrophobic core of the bilayer.

The density peaks representing the seven helices have been interpreted according to the previous assignment of sequence segment ${ }^{17}$ to the projection map of bovine rhodopsin. ${ }^{16}$ The centres of the peaks on section $+12 \AA$ and section $-8 \AA$ (Fig. 1B) were used to calculate tilt angles for seven transmembrane helices. These tilt angles, which ignore possible helix curvature or kinks but give an indication of the tilt direction for each helix, are shown in Table 1. The density assigned to helix 3 has an inclination of $30 \AA$ and is the most tilted of the helices. Helix 3 is buried inside the molecule. Its central position results in extensive contact with helices $2,4,5$ and 6 on the intracellular side and with 2, 4 and 7 towards the intradiscal side. Helix 3 closes the binding pocket of the retinal towards the cytoplasmic side and it holds the vertical helices 4, 6 and 7 apart in the part of the structure that is closer to the intracellular surface. Currently, it is not possibly to establish the precise starting and end points of the helices because of the limited vertical resolution. Accordingly the density that continues on the path of helix 3 might either represent a continuation of helix 3 outside the hydrophobic core of the bilayer or be part of the third intracellular loop connecting helices 5 and 6. Additional density observed towards the intradiscal end of helix 3 could be part of the loop connecting helices 4 and 5 . This loop must reach between the extracellular ends of the helices to form the disulphide bond (C110-C187) ${ }^{20}$ at the extracellular end of helix 3.

The density assigned to helix 4 is the least tilted feature and appears to be the shortest helix in the structure. It is separated from helices 6 and 7 by helices 2 and 3 . This produces a three-layered arrangement of the helices in the intracellular half of the molecule which interacts with transducin.

Helix 5 is not so well resolved. We see helix 5 as a tilted feature $\left(23^{\circ}\right)$ sloping from the bottom of helix 4 . As helix 5 ascends towards the intracellular side it appears to merge with helix 3 roughly $16 \AA$ above the middle of the lipid bilayer.

Helix 6 is oriented nearly perpendicular to the membrane plane in the cytoplasmic half of the molecule. However, helix 6 appears bent towards helix 5 closer to the intradiscal side. This bend allows helix 6 to maintain contact with helix 5 and prevents the interior from becoming exposed to the lipid bilayer.

Helix 7 is assigned to a feature in the map oriented almost perpendicular to the plane of the membrane. It is close to helix 3 in the centre of the molecule above the probable region where the retinal is likely to be attached. Near the intradiscal side close to the Schiff base helix 7 appears to be disorted.

\section{Comparison of rhodopsin with bacteriorhodopsin}

Bacteriorhodopsin is a light-driven proton pump found in salt-loving archaebacteria. Like rhodopsin it is a seven transmembrane helix protein. It has an all-trans retinal bound via a protonated Schiff base to the $\epsilon$-amino group of lysine (K261) in helix 7. There is no significant

Table 1. Estimate of the axes of the seven helices in rhodopsin

\begin{tabular}{lrrrrr}
\hline & \multicolumn{2}{c}{ Orientation } & & \multicolumn{2}{c}{ Position } \\
\cline { 2 - 3 } \cline { 5 - 6 } Helix & $\begin{array}{c}\text { Theta } \\
(\text { deg })\end{array}$ & $\begin{array}{c}\text { Phi } \\
(\mathrm{deg})\end{array}$ & & $\begin{array}{c}x_{0} \\
(\AA)\end{array}$ & $\begin{array}{c}y_{\mathrm{o}} \\
(\AA)\end{array}$ \\
\hline 1 & 28.4 & 141.0 & & -2.16 & 7.52 \\
2 & 27.2 & 82.2 & & -6.24 & 15.08 \\
3 & 29.6 & 50.7 & & -1.92 & 23.92 \\
4 & 3.8 & 116.6 & & -7.04 & 30.08 \\
5 & 22.7 & -11.0 & & 5.08 & 34.56 \\
6 & 7.4 & -90.0 & & 10.40 & 23.16 \\
7 & 13.4 & -165.4 & & 6.36 & 15.32 \\
\hline
\end{tabular}

The axes are determined from the coordinates of the observed peaks on $z$-sections $-8 \AA$ and $+12 \AA$ of the rhodopsin map. ${ }^{19}$ The crystallographic $\mathbf{b}$ axis is horizontal in Fig. 1B, and the $\mathbf{c}$ axis is perpendicular to the plane of the membrane, with $+\mathbf{c}(+\mathbf{Z})$ pointing towards the intracellular side. Since the crystallographic axes are not orthogonal, coordinates are referred to orthogonal axes $\mathbf{X}$ and $\mathbf{Y}$, where $\mathbf{Y}$ is parallel to $\mathbf{b}$ and $\mathbf{X}$ is perpendicular to $\mathbf{b}$ and $\mathbf{c}$. Theta is the angle between the helix and the direction of the $+\mathbf{Z}$ axis; $P$ hi is the angle around the $+\mathbf{Z}$ axis measured from the direction of the $+\mathbf{Z}$ axis; a positive phi angle indicates a right-handed rotation about $+\mathbf{Z}$ (i.e. positive phi towards $+\mathbf{Y}$, negative phi towards $-\mathbf{Y}$ ). $x_{0}, y_{0}$ are the coordinates in ängstroms, along the $\mathbf{X}, \mathbf{Y}$ axes, at which the helix axis intersects section $z=0$. Adapted from Unger et al. ${ }^{19}$ 

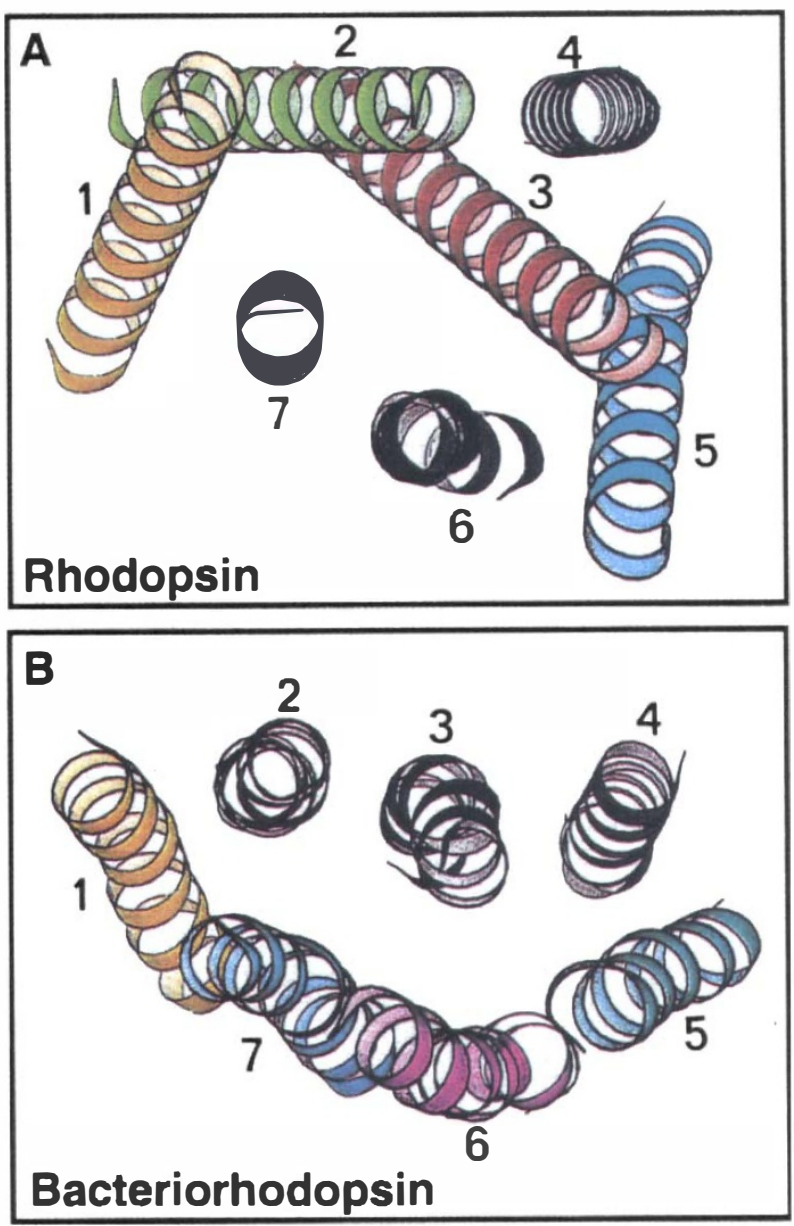

Fig. 2. The arrangement of alpha helices in rhodopsin and bacteriorhodopsin. A ribbon diagram of rhodopsin was drawn using the coordinates from a recently published alpha-carbon template for the transmembrane helices in the rhodopsin family of G-protein-coupled receptors. ${ }^{21}$ A similar diagram for bacteriorhodopsin was drawn with the coordinates from the bacteriorhodopsin structure. ${ }^{37}$ The diagrams illustrate the different arrangements of helices in bacteriorhodopsin and rhodopsin.

sequence similarity detectable between the two retinal proteins. The structure of bacteriorhodopsin has been determined by electron crystallography to near-atomic resolution. ${ }^{6}$ Ribbon diagrams of rhodopsin and bacteriorhodopsin illustrate the different arrangements of helices in the two molecules (Fig. 2).

Bacteriorhodopsin consists of three helices nearly perpendicular to the membrane plane (helices 2, 3 and 4) arranged parallel to a row of tilted helices (helices 1, 7,6 and 5). In rhodopsin a band of tilted helices (helices 1, 2, 3 and 5) runs through the middle of the molecule with two nearly perpendicular helices on one side (helices 7, 6 ) and one helix on the other side (helix 4). Angles between pairs of helices have been calculated from the helix axes and they are compared in Table 2 . The significantly different angles between pairs of helices $2^{\wedge} 3$, $3^{\wedge} 4,5^{\wedge} 6,7^{\wedge} 1,2^{\wedge} 7$ and $3^{\wedge} 6$ are indicative of the different packing of helices in rhodopsin and bacteriorhodopsin. They are independent of the overall orientation of either molecule in the membrane. Therefore mammalian rhodopsin appears to be a better model for G-protein- coupled receptors than archaebacterial

bacteriorhodopsin.

\section{The retinal binding site in rhodopsin}

The 11-cis retinal binds via a Schiff base linkage to the $\epsilon$-amino group of a lysine residue $\mathrm{K} 296$ in helix 7. The normal counter-ion for the protonated Schiff base is a glutamic acid residue E113 in helix 3 and the interaction might be mediated by a water molecule. ${ }^{21}$ Inspection of the rhodopsin structure shows that the helices are closely packed on the intracellular side of the molecule. The tilted helices 2 and 3 pack between the more perpendicular helices 4,6 and 7, forming a three-layered structure (Fig. 1B, section $+12 \AA$ ). The arrangement opens up towards the extracellular side forming a cavity that serves as the binding pocket for the retinal. ${ }^{22,23}$ It is formed by helices 3, 4, 5, 6 and 7 (Fig. 1, section $-8 \AA$ ). This cavity is closed towards the intracellular side by the long and highly tilted helix 3 , and must be closed towards the extracellular side by the loop linking helices 4 and 5 which is linked by a disulphide (C110-C187) to the extracellular end of helix $3 .^{20}$ The retinal binding site is closer to the extracellular side of the molecule. The retinal chromophore lies at an angle of about $16^{\circ}$ to the plane of the membrane. ${ }^{24}$ The retinal is more likely to point towards the intracellular side from the Schiff base. This places the $\beta$-ionon ring of the retinal close to the conserved tryptophan W265 and the retinal polyene chain would be close to glycine G121 in helix $3 .^{22,23}$

\section{The intracellular G-protein binding region}

Less density is observed on the cytoplasmic side in comparison with the extracellular side, suggesting that the cytoplasmic loops are more loosely packed than the

Table 2. Angles between pairs of helices of rhodopsin and bacteriorhodopsin

\begin{tabular}{lcc}
\hline Helix pair & $\begin{array}{c}\text { Rhodopsin } \\
\text { omega } \\
\text { (deg) }\end{array}$ & $\begin{array}{c}\text { Bacteriorhodopsin } \\
\text { omega } \\
\text { (deg) }\end{array}$ \\
\hline $7^{\wedge} 1$ & 22.9 & 8.2 \\
$1^{\wedge} 2$ & 26.5 & 27.0 \\
$2^{\wedge} 3$ & 15.0 & 5.6 \\
$3^{\wedge} 4$ & -28.2 & -10.1 \\
$4^{\wedge} 5$ & 25.2 & 20.3 \\
$5^{\wedge} 6$ & 22.4 & 7.7 \\
$6^{\wedge} 7$ & 13.5 & 12.8 \\
$2^{\wedge} 7$ & 34.4 & 21.2 \\
$3^{\wedge} 6$ & 35.6 & 18.2 \\
\hline
\end{tabular}

The angles between helix pairs were computed to compare the arrangements of helices in the seven helix retinal proteins rhodopsin and bacteriorhodopsin. Omega is the angle between the pair of helix axes specified. The omega angles are compared with those for the helix axes in bacteriorhodopsin, computed from the data of Havelka et al. ${ }^{36}$ The significantly different angles for $7^{\wedge} 1,2^{\wedge} 3,3^{\wedge} 4,5^{\wedge} 6,2^{\wedge} 7$ and $3^{\wedge} 6$ are indicative of the different packing of the helices in rhodopsin and bacteriorhodopsin. Positive angles indicate a left-handed relationship and negative numbers a right-handed relationship. 
extracellular loops. The furthest extension of density on the cytoplasmic side is that corresponding to helix 6 . Evidence to support this comes from site-directed EPR measurements. ${ }^{25}$ Portions of the third cytoplasmic loop have been functionally implicated in interactions with transducin, ${ }^{26}$ arrestin $^{27}$ and rhodopsin kinase. ${ }^{28-30}$ However, large segments of the second and third cytoplasmic loops can be deleted without affecting the ground state structure of the protein, as reflected by its ability to bind retinal. ${ }^{31}$

The helix arrangement close to the cytoplasmic surface of rhodopsin is significantly more compact near the extracellular surface (compare section $+12 \AA$ with section $-8 \AA$ ). The formation of photoactivated rhodopsin (metarhodopsin II) was shown to be associated with the movement of helices. ${ }^{32-34}$ If this movement expands the cytoplasmic surface area, it would be consistent with the observation that formation of metarhodopsin II occurs with an increase in the overall volume of rhodopsin ${ }^{35}$ and provides a newly available binding site for transducin. ${ }^{26}$

\section{Conclusions}

The arrangement of the alpha-helices in rhodopsin has been determined by low-dose electron cryomicroscopy. The structure of rhodopsin is similar to that of bacteriorhodopsin around the retinal binding pocket, which is closer to the extracellular side of the molecule. However, near the intracellular side, which interacts with the G-protein transducin, we observed three layers of helices arranged differently from bacteriorhodopsin. This arrangement changes after photoactivation and is thought to provide the G-protein binding site.

Movements of helices are thought to transmit changes caused by the isomerisation of 11-cis retinal in the retinal binding pocket to the intracellular surface of the rhodopsin molecule. This conformational change triggers the visual cascade. Recently, we have obtained betterordered crystals of rhodopsin, and with improved image processing methods we hope to obtain higher-resolution structures of rhodopsin and a structure of the photoactivated state, metarhodopsin II.

I would like to thank Joyce Baldwin for providing her model coordinates, Claudio Villa for support in crystallising rhodopsin and preparing illustrations and Patricia Edwards for support with electron microscopy and reading the manuscript. I would also like to thank Vinzenz Unger and Angelika Krebs for image processing, and Paul Hargrave and Hugh McDowell for help with crystallising frog rhodopsin. Richard Henderson deserves special thanks for help with the MRC image processing programs, long-term support and encouragement.

\section{References}

1. Stadel JM, Wilson S, Bergsma DJ. Orphan G protein-coupled receptors: a neglected opportunity for pioneer drug discovery. Trends Pharmacol Sci 1997;18:430-7.

2. Khorana HG. Rhodopsin, photoreceptor of the rod cell: an emerging pattern for structure and function. J Biol Chem 1992;267:1-4.
3. Hargrave PA, McDowell JH. Rhodopsin and phototransduction. Int Rev Cytol 1993;137B:49-97.

4. Deisenhofer H, Epp O, Miki K, Huber R, Michel H. Structure of the protein subunits in the photosynthetic reaction center of Rhodopseudomonas viridis at $3 \AA$ resolution. Nature 1985;318:618-24.

5. Weiss M, Kreusch A, Schiltz E, Nestal U, Welte W, Weckesser J, Schulz GE. The structure of porin from Rhodobacter capsulatus at $1.8 \AA$ resolution. FEBS Lett 1991;280:379-82.

6. Henderson R, Baldwin J, Ceska T, Zemlin F, Beckmann E, Downing K. Model for the structure of bacteriorhodopsin based on high-resolution electron cryo-microscopy. J Mol Biol 1990;213:899-929.

7. Kühlbrandt W, Wang DN, Fujiyoshi Y. Atomic model of plant light-harvesting complex by electron crystallography. Nature 1994;367:614-21.

8. Boettcher B, Wynne SA, Crowther RA. Determination of the fold of the core protein of hepatitis B virus by electron cryomicroscopy. Nature 1997;386:88-91.

9. Unwin N. Acetylcholine-receptor channel imaged in the open state. Nature 1995;373:37-43.

10. Toyoshima C, Sasabe H, Stokes DL. Three-dimensional cryoelectron microscopy of the calcium-ion pump in the sarcoplasmic reticulum membrane. Nature 1993;362:469-71.

11. Crowther RA, Henderson R, Smith JM. MRC image processing programs. J Struct Biol 1996;116:9-16.

12. Kühlbrandt W. Two-dimensional crystallization of membrane proteins. Q Rev Biophys 1992;25:1-49.

13. Mitchell DC, Lawrence JTR, Litman BJ. Primary alcohols modulate the activation of the G-protein-coupled receptor rhodopsin by a lipid-mediated mechanism. J Biol Chem 1996;271:19033-6.

14. Corless JM, McCaslin DR, Scott BL. Two-dimensional rhodopsin crystals from disk membranes of frog retinal outer segments. Proc Natl Acad Sci USA 1982;79:1116-20.

15. Schertler GFX, Hargrave PA. Projection structure of frog rhodopsin in two crystal forms. Proc Natl Acad Sci USA 1995;92:11578-82.

16. Schertler GFX, Villa C, Henderson R. Projection structure of rhodopsin. Nature 1993;362:770-2.

17. Baldwin JM. The probable arrangement of the helices in G-protein-coupled receptors. EMBO J 1993;12:1693-703.

18. Unger VM, Schertler GFX. Low resolution structure of bovine rhodopsin determined by electron cryo-microscopy. Biophys J 1995;68:1776-86.

19. Unger VM, Hargrave PA, Baldwin JM, Schertler GFX. Arrangement of rhodopsin transmembrane alpha helices. Nature 1997;389:203-6.

20. Karnik SS, Khorana HG. Assembly of functional rhodopsin requires a disulfide bond between cysteine residues 110 and 187. J Biol Chem 1990;265:17520-4.

21. Baldwin JM, Schertler GFX, Unger VM. An alpha-carbon template for the transmembrane helices in the rhodopsin family of G-protein-coupled receptors. J Mol Biol 1997;272:144-64.

22. Han M, Smith SO. High-resolution structural studies of the retinal-Glu113 interaction in rhodopsin. Biophys Chem 1995;56:23-9.

23. Zhang $\mathrm{HZ}$, et al. The location of the chromophore in rhodopsin: a photoaffinity study. J Am Chem Soc 1994;116:10165-73.

24. Liebman PA. In situ microspectrophotometric studies on the pigments of single retinal rods. Biophys J 1962;2:61-78.

25. Altenbach C, Yang K, Farrens DL, Farahbakhsh ZT, Khorana HG, Hubbell WL. Structural features and light-dependent changes in the cytoplasmic interhelical E-F loop region of rhodopsin: a site-directed spin-labelling study. Biochemistry 1996;35:12470-8.

26. König B, Arendt A, McDowell JH, Kahlert M, Hargrave PA, Hofmann KP. Three cytoplasmic loops of rhodopsin interact with transducin. Proc Natl Acad Sci USA 1989;86:6878-82. 
27. Krupnick JG, Gurevich VV, Schepers T, Hamm HE, Benovic JL. Arrestin-rhodopsin interaction: multi-site binding delineated by peptide inhibition. J Biol Chem 1994;269:3226-32.

28. Wilden U, Hall SW, Kühn H. Phosphodiesterase activation by photoexcited rhodopsin is quenched when rhodopsin is phosphorylated and binds the intrinsic $48-\mathrm{kDa}$ protein of rod outer segments. Proc Natl Acad Sci USA 1986;83:1174-8.

29. Palczewski K, Buczylko J, Kaplan MW, Polans AS, Crabb JW. Mechanism of rhodopsin kinase activation. J Biol Chem 1991;266:12949-55.

30. Thurmond RL, Creuznet C, Reeves PJ, Khorana HG. Structure and function in rhodopsin: peptide sequences in the cytoplasmic loops of rhodopsin are intimately involved in interaction with rhodopsin kinase. Proc Natl Acad Sci USA 1997;94:1715-20.

31. Franke RR, Sakmar TP, Graham RM, Khorana HG. Structure and function in rhodopsin: studies of the interaction between the rhodopsin cytoplasmic domain and transducin. J Biol Chem 1992;267:14767-74.
32. Farrens DL, Altenbach C, Yang K, Hubbell WL, Khorana HG. Requirement of rigid-body motion of transmembrane helices for light activation of rhodopsin. Science 1996;274:768-70.

33. Sheikh SP, Zvyaga TA, Lichtarge O, Sakmar TP, Bourne HR Rhodopsin activation blocked by metal-ion-binding sites linking transmembrane helices $C$ and F. Nature 1996;383:347-50.

34. Farahbaksh ZT, Ridge KD, Khorana HG, Hubbell WL. Mapping light-dependent structural changes in the cytoplasmic loop connecting helices C and D in rhodopsin: a site-directed spin labelling study. Biochemistry 1995;34:8812-9.

35. Lamola AA, Yamane T, Zipp A. Effects of detergents and high pressures upon the metarhodopsin $\mathrm{I} \Leftrightarrow$ metarhodopsin II equilibrium. Biochemistry 1974;13:738-45.

36. Havelka WA, Henderson R, Oesterhelt D. Three-dimensional structure of halorhodopsin at $7 \AA$ resolution. J Mol Biol 1995;247:726-38.

37. Grigorieff N, Ceska TA, Downing KH, Baldwin JM, Henderson R. Electron-crystallographic refinement of the structure of bacteriorhodopsin. J Mol Biol 1996;259:393-421. 